\title{
Genetics - Need to Evaluate its Role in Early Detection of Oral Squamous Cell Carcinoma (OSCC)
}

\author{
Urvashi Singla, Snehlata Kulhari
}

\section{ABSTRACT}

The concept of cancer genetics is relatively new and considered more in prostate and breast cancer. Its role in OSCC has never been given much attention, which is the sixth most common malignancy worldwide. It is a tumour which can be treated by simple surgical procedure, if diagnosed at an early stage. Oral cavity and oropharynx are easily accessible for visualization. But high prevalence of oral abnormalities (5-15\%) detected as a result of oral screening programmes (OSP) subjects large number of patients for biopsy which is impractical. Detection of early stage oral cancer is significantly impaired by absence of classic clinical characteristics of advanced oral cancer in early stage lesions. This influences 5 year survival rate for $80 \%$ of patients, despite major advances in surgery, chemotherapy, radiotherapy.

Many gene alterations have been implicated in development and progression of OSCC. Genetic differences is an important consideration to determine individual differences in susceptibility to chemical carcinogens. Many genetic changes are commonly observed in oral cancers. Salivary Transcriptome Diagnostics, based on specific mRNA signature in saliva as diagnostic marker, can be a suitable tool for development of non invasive diagnostic, chemoprevention, prognostic and follow up tests for cancer and also to detect cancer conversion of premalignant lesions. To summarize research on genetics will open new directions for estimation of risk of human cancer and its early diagnosis.

Key Words: Genetics; OSCC; Salivary Transcriptome Diagnostics; Chemoprevention; Prognosis

\section{Contact Author}

Dr. Urvashi Singla

E-mail : snehkulhari@yahoo.co.in
- Cancer genetics is a field that study type of mutations as well as consequences of these in cancer cells

- Two major classes of cancer genes are:(1)

- That directly affect cell growth either positively (oncogenes) or negatively (tumour suppressor genes)

- That affect the ability of cell to maintain the integrity of its genome

- OSCC is frequently affected by expression of genes involved in:

- DNA repair

- Stability of genome

\section{GENETICS - Role in development and} progression of OSCC

- It is important to understand the oral cancer molecular biology and genetics to study its role for diagnosis of OSCC

- Deregulation of molecular mechanism controlling cell cycle progression is hallmark of cancer

- Genetic changes observed in oral cancer are(2)

1. Loss of heterozygosity $(\mathrm{LOH})$ at suspected tumour suppressor genes : 
- 3p (FHIT).

9p (CDKN2A)

- 17p (Tp53)

2. Gene amplification and over expression of particular oncogenes.

3. Mutation in Ras gene

- Frequently in India

- Especially in cancer linked with tobacco chewing.

4. Telomerase activation

- Promotes survival of cells carrying genetic abnormality.

5. Over expression of COX-2 and pEGFR.

- Useful molecular target for treatment.

6. Polymorphism in genes involved in metabolism of carcinogens in tobacco and alcohol.

- Linked to individual susceptibility.

- Null genotype for GSTM1 and GSTT1 have increase risk for cancer.

- CYP450, N-acetyl transferase, alcohol dehydro-genase - play a role but not clear yet.

7. Increase risk with number of inherited cancer syndromes :

- Li-Fraumeni

- Fanconi anaemia

- Xeroderma pigmentosum

8. $\mathrm{p}^{16 \mathrm{INK} 4 \mathrm{~A}}$ expression:(3)

- Independent prognostic indicator

- High chemotherapeutic sensitivity

- No lymphnode metastasis

\section{GENETICS - Role in early detection of OSCC}

- OSCC is the sixth most common malignancy worldwide.(4)

- Can be treated by simple surgical procedure, if diagnosed at early stage.

- Detection of early stage cancer is significantly impaired by absence of classic clinical characteristics of advanced oral cancer in early stage lesions.

- Ulceration

- Induration

- Elevation

- Bleeding

- Cervical adenopathy

- Genetics evaluation can be helpful in early diagnosis of OSCC.

- Genetic differences is important consideration to determine individual differences in susceptibility to chemical carcinogens.

- Salivary transcriptome diagnostics, based on specific mRNA signature in saliva as diagnostic marker, can be a suitable tool for development of non-invasive, diagnostic, chemoprevention, prognostic and follow up test for cancer and also to detect cancer conversion of premalignant lesions.

- Sources of salivary RNA

1. Salivary gland:

- Parotid
- Submandibular

- Sublingual

- Minor glands

2. GCF (Gingival crevicular fluid)

3. Oral mucosal cells:

- Lining

- Desquamated

- Cancer associated genes upregulated in saliva from oral cancer patients:

- IL8 (Interleukin 8)

- IL1B (Interleukin 1-Beta)

- Ferritin polypeptide mRNA.

- OAZ1 (Ornithine decarboxylase antizyme 1)

- SAT (Spermidine N1-acetyl transferase)

\section{GENETICS - Role in prevention and control of OSCC}

- Cancer can be prevented or controlled through interference with factors which cause initiation, promotion or progression

- Smoking cessation does not markedly decrease the cured patients risk of second malignancy (5)

- It may halt the early stages of carcinogenesis (metaplasia), but has no effect on late stages

- Thus, work has to be done on genetics for prevention and control of OSCC

- Chromosomal alterations provide important clue to genetic changes in cancer and can lead to mechanical based design and development of new generation of cancer drugs

- Knowledge of molecular events governing cell cycle regulation, has lead to development of Oncolytic viruses, designed to replicate in tumour cells (which lack p53 pathway)

- These are Adenoviruses mutant lacking viral p55 protein and has demonstrated efficacy especially when combined with 5-Fluorouracil and cisplatin(1)

\section{Summary and Conclusion}

Cancer develops through an interaction between genetic and environmental exposures. Much work has been done on its prevention and control, with no stress on early detection. Primary prevention concerns the identification and manipulation of genetic, biologic and environmental factors in casual pathway. Secondary prevention concerns identification of asymptomatic neoplastic lesions combined with effective therapy. But, detecting cancer in asymptomatic individuals can save lives. A number of genes have been identified that predispose for cancer. Testing for these genes can define a high risk population, which are ideal candidates for chemoprevention and intensive screening. Thus, primary and secondary prevention, if combined with early detection, can be effective tool to decrease morbidity and mortality and to improve diagnosis and prognosis of OSCC. 


\section{THE AUTHORS}

\section{Dr. Urvashi Singla}

MDS (Oral Surgery)

Associate Professor \& Head,

Deptt. of Oral \& Maxillofacial Surgery, Government Dental College \& Hospital, Patiala (Punjab).

Address for correspondence:

Dr. Urvashi Singla

109, Hem Bagh, Kheri Road, Patiala.

Ph. 0175-2228878

\section{Dr. Snehlata Kulhari}

BDS Intern

Govt. Dental College \& Hospital,

Patiala (Punjab)

E-mail: snehkulhari@yahoo.co.in

\section{References}

1. Harrisons Principle of Internal Medicine.Kasper et al.16 $6^{\text {th }}$ edition. Volume 1. The Mc Graw Hill companies, Part V, Oncology and Hematology: Neoplastic Disorders (section 1): Cancer Genetics 447-452.

2. Polymorphisms of the CYP1A1 and GSTM1 gene involved in oral squamous cell carcinoma in association with a cigarette dose. Tanimoto K, Hayashi S, Yoshiga K, Ichikawa T. Oral Oncol 1999;35(2):191-6.

3. P16 $16^{\mathrm{INKA}}$ in Oral Squamous Cell Carcinomas - A Correlation With Biological Behaviors: Immunohistochemical and FISH Analysis Suzuki H, Sugimura H, Hashimoto K. Journal of Oral Maxillofacial Surgery 2006;64:1617-1623

4. Does administration of isosorbide mononitrate affect cellular proliferation in oral squamous cell carcinoma? A prospective randomized clinical study. Downie IP, Umar T, Boote DJ, Mellor TK, Hoffman GR, Brennan PA. Journal of Oral Maxillofacial Surgery 2004;62:1064-1068.

5. Harrisons Principle of Internal Medicine.Kasper et al.16 ${ }^{\text {th }}$ edition. Volume 1The Mc Graw Hill companies, Part V, Oncology and Hematology: Neoplastic Disorders (section 1) Prevention and early detection of cancer 441-446. 